\title{
Electrospun fibrous scaffolds combined with nanoscale hydroxyapatite induce osteogenic differentiation of human periodontal ligament cells
}

This article was published in the following Dove Press journal:

International Journal of Nanomedicine

27 August 2014

Number of times this article has been viewed

\section{Xiaonan $\mathrm{Wu}^{\mathrm{I}}$ \\ Leiying Miao,\# \\ Yingfang $\mathrm{YaO}^{3}$ \\ Wenlei Wu' \\ Yu Liu' \\ Xiaofeng Chen' \\ Weibin Sun ${ }^{1, \#}$}

'Department of Periodontology, Hospital of Stomatology, Medical School of Nanjing University, Nanjing, People's Republic of China;

${ }^{2}$ Department of Cariology and Endodontics, Hospital of Stomatology, Medical School of Nanjing University, Nanjing, People's Republic of China; ${ }^{3}$ Eco-materials and Renewable Energy Research Center, Department of Materials Science and Engineering, National Laboratory of Solid State Microstructures, Nanjing University, Nanjing, People's Republic of China

${ }^{\#}$ These authors contributed equally to this work

Correspondence: Weibin Sun Department of Periodontology, Hospital of Stomatology, Medical School of Nanjing University, 30 Zhongyang Road, Nanjing 210008, People's

Republic of China

Tel +862583620173

Fax +862583620173

Email wbsun@nju.edu.cn

Leiying Miao

Department of Cariology and Endodontics, Hospital of Stomatology, Medical School of Nanjing University,

30 Zhongyang Road, Nanjing, 210008,

People's Republic of China

Tel +862583620173

Fax +862583620173

Email miaoleiying80@163.com
Abstract: Periodontal repair is a complex process in which regeneration of alveolar bone is a vital component. The aim of this study was to develop a biodegradable scaffold with good biocompatibility and osteoinductive ability. Two types of composite fibrous scaffolds were produced by electrospinning, ie, type I collagen/poly( $\varepsilon$-caprolactone) (COL/PCL) and type I collagen/poly( $\varepsilon$-caprolactone)/nanoscale hydroxyapatite (COL/PCL/nHA) with an average fiber diameter of about $377 \mathrm{~nm}$. After a simulated body fluid (SBF) immersion test, the COL/PCL/ nHA-SBF scaffold developed a rough surface because of the calcium phosphate deposited on the fibers, suggesting that the presence of nHA promoted the mineralization potential of the scaffold. Energy dispersive X-ray spectroscopy clearly showed the calcium and phosphorus content in the COL/PCL/nHA and COL/PCL/nHA-SBF scaffolds, confirming the findings of nHA and calcium phosphate precipitation on scanning electron micrographs. Water contact analysis revealed that nHA could improve the hydrophilic nature of the COL/PCL/nHA-SBF scaffold. The morphology of periodontal ligament cells cultured on COL/PCL-SBF and COL/ PCL/nHA-SBF was evaluated by scanning electron microscopy. The results showed that cells adhered to either type of scaffold and were slightly spindle-shaped in the beginning, then extended gradually with stretched filopodia, indicating an ability to fill the fiber pores. A Cell Counting Kit-8 assay showed that both scaffolds supported cell proliferation. However, real-time quantitative polymerase chain reaction analysis showed that expression of the bone-related markers, alkaline phosphatase and osteocalcin, was upregulated only on the COL/PCL/nHA-SBF scaffold, indicating that this scaffold had the ability to induce osteogenic differentiation of periodontal ligament cells. In this study, COL/PCL/nHA-SBF produced by electrospinning followed by biomimetic mineralization had combined electrospun fibers with nHA in it. This scaffold has good biocompatibility and osteoinductive ability as a result of the characteristics of nHA, so could be innovatively applied to periodontal tissue engineering as a potential scaffold.

Keywords: nanoscale hydroxyapatite, electrospinning, periodontal ligament cells

\section{Introduction}

Periodontitis is a common chronic inflammatory disease. Periodontal defects resulting from periodontitis include significant destruction of alveolar bone and the periodontal ligament tissue, root cementum, and gingiva, eventually leading to loss of teeth if untreated. Ideal treatment should involve not only the prevention of further attachment loss, but regeneration of the destroyed tissues. Periodontal repair is a complex process in which regeneration of osseous tissue is a vital component. ${ }^{1-5}$

Many bone or bone substitutes have been used in debrided periodontal defects with the aim of promoting alveolar bone regeneration. The types of grafts utilized include autogenous grafts (derived from the same individual), allogenic grafts (derived from 
a different member of the same species), xenografts (derived from different species), and alloplastic materials (synthetic products). Hydroxyapatite (HA) has a stoichiometry similar to that of bone mineral and has been used extensively as a bone substitute in the People's Republic of China and abroad for several decades. ${ }^{6}$ A number of successful cases have demonstrated that HA is not cytotoxic, is nonirritant, has good biological activity, is highly biocompatible, and has stable physicochemical properties. However, HA also has some disadvantages, in that it is brittle and has low fracture strength, which makes it unsuitable for application in loadbearing areas. Further, the osteoinductivity that HA does not have is a very important condition for bone regeneration. The abovementioned problems with HA have already limited its clinical application to some extent..$^{7-9}$

Bone is mostly made up of a composite material incorporating nanoscale hydroxyapatite $(\mathrm{nHA})\left[\mathrm{Ca}_{10}\left(\mathrm{PO}_{4}\right)_{6}(\mathrm{OH})_{2}\right]$ and collagen fibers. ${ }^{10} \mathrm{nHA}$ deposited parallel to collagen fibers forms an organic-inorganic nanometer compound structure that confers provide rigidity and tenacity simultaneously. ${ }^{11,12}$ Therefore, nHA is more similar in morphology and crystal structure to the apatite found in natural bone or teeth than conventional regimens, and has better biological properties. ${ }^{13}$ Our previous work has demonstrated that nHA had the ability to induce osteogenic differentiation of periodontal ligament cells (PDLCs) and may thus be a suitable tissue engineering scaffold for intrabony periodontal defects. ${ }^{14}$ Following on from this finding, we undertook the present study, in which we embedded nHA in collagen fibers using electrospinning technology to mimic natural bone structure.

Electrospinning is a technique that uses electrostatic forces to produce fine fibers from polymer solutions or melts. The fibers produced by this spinning method have a thinner diameter (from nanometer to micrometer) and a larger surface area than those obtained by conventional spinning processes. ${ }^{15,16}$ Fibers prepared by this technique have shown the potential to mimic extracellular matrix in terms of both morphology and composition, and thus may facilitate cell attachment and growth. ${ }^{17}$ Electrospun fibers are used widely in biomedical applications (as tissue engineering scaffolds or biomimetic substitutes), wound healing, and drug delivery, with further applications being the subject of ongoing research. ${ }^{18,19}$

In this study, we used electrospinning followed by biomimetic mineralization to produce fibers with a structure conducive to the proliferation, metabolism, and osteogenic differentiation of human PDLCs. This fibrous scaffold might be innovatively applied in periodontal tissue engineering.

\section{Materials and methods}

\section{Fabrication of scaffolds}

Via the electrospinning technique, type I collagen (COL; Sigma-Aldrich, Wadena, MN, USA), poly( $\varepsilon$-caprolactone) (PCL, molecular weight 160 kDa; Dai Gang Biology, Shanghai, People's Republic of China), and nHA (size $<100 \mathrm{~nm}$; Aladdin, Shanghai, People's Republic of China) were utilized to prepare two kinds of scaffolds, ie, type I collagen/ poly( $\varepsilon$-caprolactone) (COL/PCL) and type I collagen/ poly( $\varepsilon$-caprolactone)/nanoscale hydroxyapatite (COL/PCL/ nHA). The solute was dissolved in hexafluoroisopropanol (Aladdin) and the composition details are listed in Table 1. A defined amount of genipin (Shifeng Biological Technology, Shanghai, People's Republic of China) was added to cross-link the collagen before electrospinning. The solution was drawn into a $5 \mathrm{~mL}$ syringe fitted with a blunt tip capillary tube (inner diameter $0.34 \mathrm{~mm}$ ). The needle-like flow of the solution from the capillary spinneret was fed by a syringe pump at a flow rate of $2.5 \mathrm{~mL}$ per hour. A high-voltage power supply was used to create voltages of $17 \mathrm{kV}$. The distance between the blunt tip capillary and the cover slips or aluminum foil used for collections was $15 \mathrm{~cm}$. After sufficient drying, the collections with electrospun fibers were stored in clean polythene bags for further use.

\section{Biomimetic mineralization}

The two scaffolds were immersed in 1.5 times simulated body fluid solution $(\mathrm{SBF} \times 1.5)$ at a temperature of $37^{\circ} \mathrm{C}$ and labeled COL/PCL-SBF and COL/PCL/nHA-SBF, respectively. $\mathrm{SBF} \times 1.5$ was prepared by dissolving $\mathrm{NaCl} 8.035 \mathrm{~g}$, $\mathrm{NaHCO}_{3} 0.355 \mathrm{~g}, \mathrm{KCL} 0.225 \mathrm{~g}, \mathrm{~K}_{2} \mathrm{HPO}_{4} \cdot 3 \mathrm{H}_{2} \mathrm{O} 0.231 \mathrm{~g}$, $\mathrm{MgCl}_{2} \cdot 6 \mathrm{H}_{2} \mathrm{O} 0.311 \mathrm{~g}, 1.0 \mathrm{M} \mathrm{HCl}(39 \mathrm{~mL}), \mathrm{CaCl}_{2} 0.292 \mathrm{~g}$, $\mathrm{Na}_{2} \mathrm{SO}_{4} 0.072 \mathrm{~g}$ and Tris $6.118 \mathrm{~g}$ in $1 \mathrm{~L}$ of MilliQ water, and the $\mathrm{pH}$ of the solution was adjusted to 7.4 with $1.0 \mathrm{M} \mathrm{HCl}^{20,21}$ After immersion for 7 days, the scaffolds were gently washed with MilliQ water and dried thoroughly.

\section{Characterizations of scaffolds}

The morphology of the COL/PCL, COL/PCL/nHA, COL/ PCL-SBF, and COL/PCL/nHA-SBF scaffolds was studied by scanning electron microscopy (Siron 200; FEI, Hillsboro,

Table I The compositions of electrospining solutions

\begin{tabular}{llllll}
\hline & HFP & COL & PCL & nHA & GP \\
\hline COL/PCL & $5 \mathrm{~mL}$ & $500 \mathrm{mg}$ & $450 \mathrm{mg}$ & $0 \mathrm{mg}$ & $6 \mathrm{mg}$ \\
COL/PCL/nHA & $5 \mathrm{~mL}$ & $500 \mathrm{mg}$ & $450 \mathrm{mg}$ & $350 \mathrm{mg}$ & $6 \mathrm{mg}$ \\
\hline
\end{tabular}

Abbreviations: HFP, hexafluoroisopropanol; COL, type-I collagen; PCL, poly ( $\varepsilon$-caprolactone); $\mathrm{nHA}$, nanoscale hydroxyapatite; GP, genipin. 
OR, USA) after sputter coating with gold (Emitech SC7620; Quorum Technologies Ltd, Ashford, UK). The elemental composition of each type of scaffold was examined by energy dispersive X-ray spectroscopy (Edax, Phoenix, AZ, USA).

The hydrophilic/hydrophobic nature of the two types of mineralized scaffold was measured using a water contact angle measuring instrument (JJ2000B2; Powereach, Shanghai, People's Republic of China). Deionized water was dropped onto the scaffolds in three different positions and average readings were taken.

\section{PDLC culture}

A first premolar tooth extracted for orthodontic reasons was placed in a $15 \mathrm{~mL}$ tube containing Dulbecco's Modified Eagle's Medium with 2\% penicillin/streptomycin. We cut the middle third of the periodontal ligament from the root surface and sectioned it into approximately $1 \times 1 \mathrm{~mm}^{2}$ pieces with a scalpel. The tissues were then put into a $25 \mathrm{~cm}^{2}$ culture flask and incubated at $37^{\circ} \mathrm{C}$ in a $5 \% \mathrm{CO}_{2}$ atmosphere for 4 hours to allow tissue adhesion. Next, $3 \mathrm{~mL}$ of Dulbecco's Modified Eagle's Medium containing 20\% fetal bovine serum and 1\% penicillin/streptomycin was gently added without dispersing the tissues. One week later, the cells started migrating outwards from the periodontal ligament tissues. The cells were passaged using $0.25 \%$ trypsin and further expanded until passage 3 .

\section{Morphology of PDLCs cultured on COL/PCL-SBF and COL/PCL/HA-SBF}

The COL/PCL-SBF and COL/PCL/HA-SBF scaffolds were placed in a 24-well plate. The samples were immersed in $75 \%$ ethanol for 30 minutes, and irradiated with ultraviolet rays for 1.5 hours. The samples were washed three times with phosphate-buffered saline and then immersed in Dulbecco's Modified Eagle's Medium before cell seeding. Cells were detached by trypsin/ethylenediaminetetraacetic acid and seeded onto the scaffolds at a density of $5 \times 10^{4}$ cells per well. Morphological evaluation of PDLCs grown on the scaffolds after 1,3 , and 8 days was performed by scanning electron microscopy.

\section{Proliferation and differentiation of PDLCs}

The COL/PCL-SBF and COL/PCL/nHA-SBF scaffolds were put in a 96-well plate and sterilized by $75 \%$ ethanol and ultraviolet rays. PDLCs were then seeded at a density of 1,000 cells per well and cultured in Dulbecco's Modified Eagle's Medium at $37^{\circ} \mathrm{C}$, with the medium exchanged every 3 days. On days 1, 3, 5, and 7, a colorimetric Cell Counting Kit-8
(Beyotime, C0038, Shanghai, People's Republic of China) was used to calculate relative cell proliferation rates. After culturing the cells in Dulbecco's Modified Eagle's Medium, they were rinsed in phosphate-buffered saline to remove unattached cells, then incubated further in Dulbecco's Modified Eagle's Medium with 10\% WST-8 for 4 hours at $37^{\circ} \mathrm{C}$. Finally, absorbance was determined at $450 \mathrm{~nm}$ using a microplate reader (SpectraMax M3 Multi-Mode; Molecular Devices, Sunnyvale, CA, USA).

To analyze the expression of osteogenic markers in PDLCs on the scaffolds, total RNA was isolated after coculturing for 10 days. Equivalent total RNA was used for synthesis of complementary DNA with a first strand cDNA synthesis kit for real-time polymerase chain reaction (PCR, PrimeScript ${ }^{\text {TM}}$; TaKaRa, Tokyo, Japan). Real-time quantitative PCR was performed on a StepOne ${ }^{\mathrm{TM}}$ real-time PCR system (Applied Biosystems, Foster City, CA, USA) using $\mathrm{SYBR}^{\circledR}$ Green Supermix (Bio-Rad Laboratories, Hercules, CA, USA). The data were analyzed by $2^{-\Delta \Delta C T}$ using StepOne software version 2.1. ${ }^{22}$ Expression of osteogenic marker genes was normalized to $\beta$-actin $\left(5^{\prime}\right.$-GAT GAG ATT GGC ATG GCT TT-3' and 5'-CAC CTT CAC CGT TCC AGT TT- $\left.3^{\prime}\right)$. The following primer sequences were used for the osteogenic marker genes: alkaline phosphatase (ALP) (5'-TGA CCA ACA TCG CTT CAG GG-3' and 5'-CCG GCT ATA TTA CCA AGA GCC C-3'); osteocalcin (5'-CAC TCC TCG CCC TAT TGG C-3' and 5'-CCC TCC TGC TTG GAC ACA AAG-3').

\section{Statistical analysis}

The statistical analysis was carried out using SigmaStat version 2.0 (SPSS Inc, Chicago, IL, USA). Unless stated otherwise, all experiments were performed in triplicate and the results are presented as the mean \pm standard deviation. Analysis of variance was used to determine statistical significance with $P<0.05$.

\section{Results}

\section{Characterization of scaffolds}

The COL/PCL scaffolds had a highly porous network, as shown in Figure 1A, with interconnected pores and a smooth surface. The COL/PCL/nHA scaffolds had a rough surface because of the nHA packed inside the fibers, with a diameter of about $377 \mathrm{~nm}$ (Figure 1B). Precipitation of calcium phosphate was visible on COL/PCL/nHA-SBF after incubation for 7 days in SBF (Figure 1D). However, for the COL/PCL-SBF composite scaffold, little precipitation of calcium phosphate could be seen on the fiber surface (Figure 1C). 

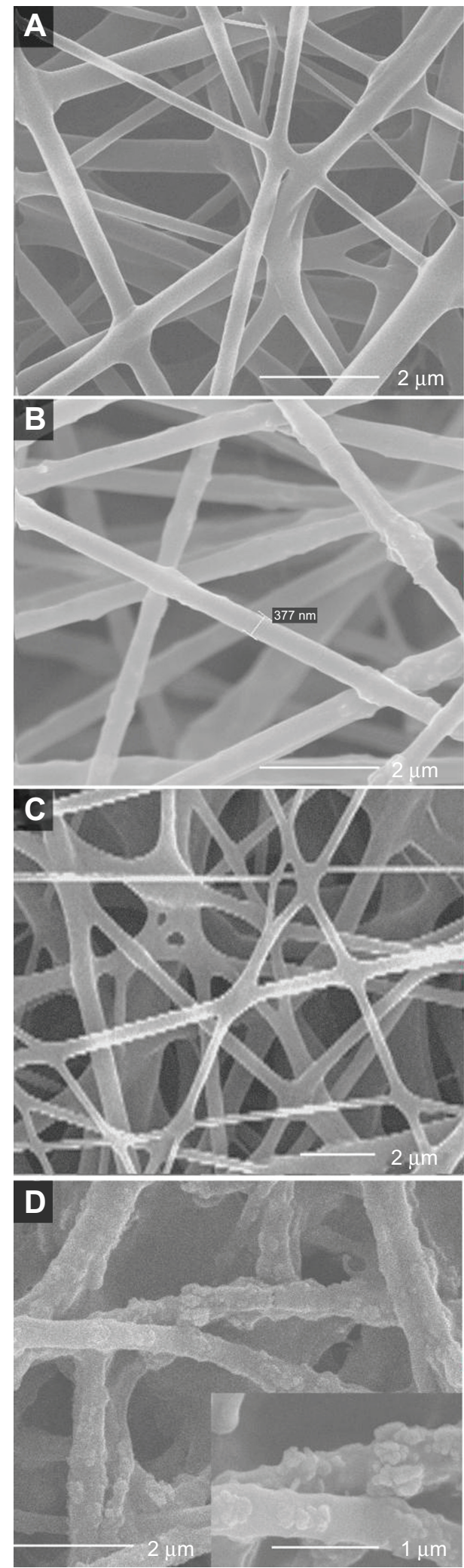
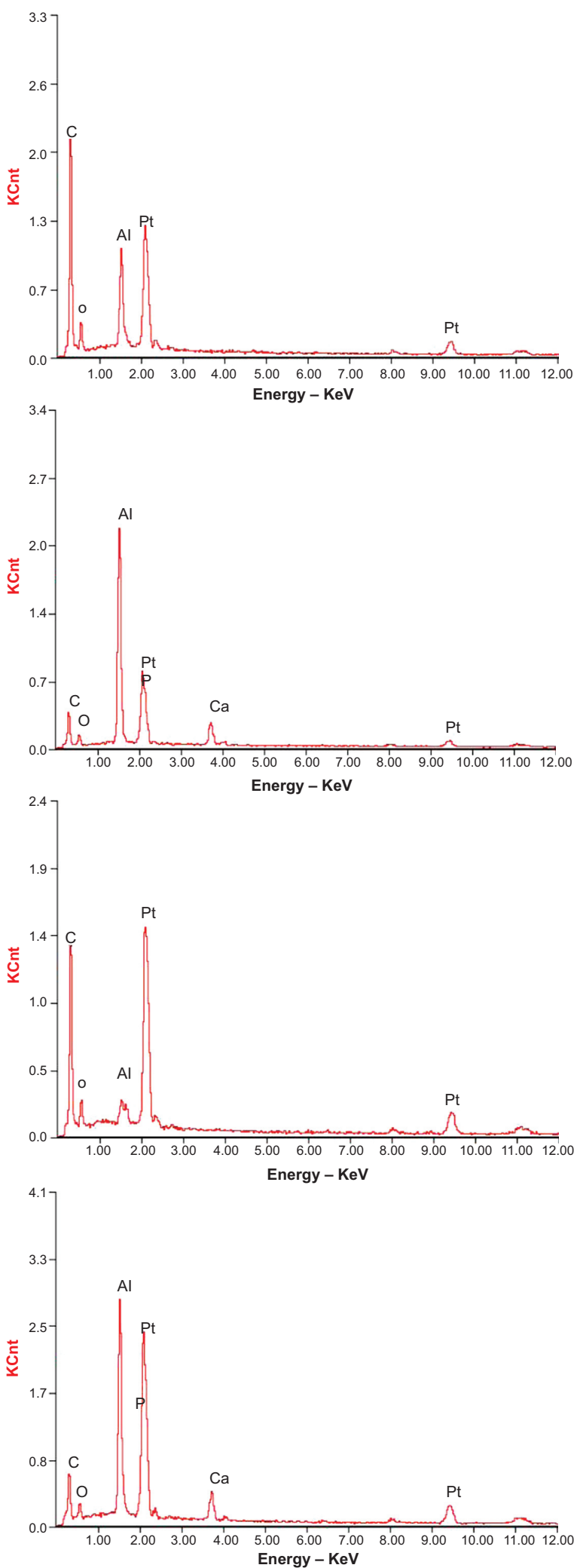

Figure I The morphology and elemental composition of the four scaffolds.

Notes: Scanning electron micrographs and their EDX spectra of the four fibrous scaffolds. (A) COL/PCL with interconnected pores and smooth surface; (B) COL/PCL/nHA with a rough surface and a diameter of about $377 \mathrm{~nm}$; (C) COL/PCL-SBF, few CaP deposits could be seen on the fiber surface; (D) COL/PCL/nHA-SBF, CaP precipitation was visible. The results of the EDX spectroscopy analysis was also consistent with SEM.

Abbreviations: SEM, scanning electron microscopy; EDX, energy dispersive X-ray spectroscopy; COL/PCL, the electrospun type-I collagen/ poly( $\varepsilon$-caprolactone) scaffold;

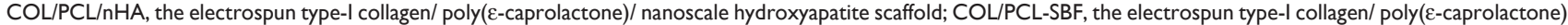
scaffold immersed in simulated body fluid; $\mathrm{COL} / \mathrm{PCL} / \mathrm{nHA}-\mathrm{SBF}$, the electrospun type-I collagen/ poly( $\varepsilon$-caprolactone)/ nanoscale hydroxyapatite scaffold immersed in simulated body fluid; CaP, calcium and phosphorus compound. 
Energy dispersive X-ray spectroscopy, an analytical technique, is used for elemental analysis. In this study, it was utilized to measure the calcium and phosphorus in the scaffolds. In contrast with the control groups, the calcium and phosphorus content on the $\mathrm{COL} / \mathrm{PCL} / \mathrm{nHA}$ and $\mathrm{COL} /$ PCL/nHA-SBF scaffolds was visible, further confirming the results for nHA and deposition of calcium phosphate seen on scanning electron microscopy (Figure 1).

The hydrophilic/hydrophobic nature of the scaffolds had a great influence on infiltration of the medium and cell adhesion. In this study, the water contact angles of COL/PCL-SBF and $\mathrm{COL} / \mathrm{PCL} / \mathrm{nHA}-\mathrm{SBF}$ decreased from $117.35^{\circ}$ to $67.43^{\circ}$, indicating an increase in hydrophilicity (Figure 2).

\section{Morphology of PDLCs cultured on COL/ PCL-SBF and COL/PCL/nHA-SBF}

After being seeded for 1,3 , and 8 days, the morphologies of PDLCs were observed by scanning electron microscopy to evaluate the cells for attachment and spreading (Figure 3). The cells adhering to the fibers were spindle-shaped (Figure 3A and D) on the first day and then found to have extended gradually and satisfactorily by day 3 (Figure 3B and E). At day 8, the PDLCs were further flattened and stretched, showing a potential to fill the fiber pores. In addition, cells on the two types of scaffolds displayed visible cilia and filopodia, reflecting their viability, with no apparent difference in morphology.

\section{Proliferation and differentiation of PDLCs on COL/PCL/nHA-SBF}

The PDLCs proliferated stably during a prolonged culture period as shown in Figure 4. At each time point, there was no obvious difference between mean values for the two groups $(P>0.05)$. Expression of ALP and osteocalcin as markers of osteogenic differentiation was analyzed by quantitative PCR. On day 10, ALP and osteocalcin expression in the experimental group were higher than that in the control group $(P<0.05$; Figure 5), indicating that the COL/PCL/nHA-SBF scaffold had the ability to induce osteogenic differentiation of PDLCs.

\section{Discussion}

The main constituents of alveolar bone are collagen ( $20 \mathrm{wt} \%)$, and calcium phosphate $(69 \mathrm{wt} \%),{ }^{11}$ with other organic materials, including proteins, polysaccharides, and lipids, also present in small quantities. Calcium phosphate, mostly in the form of crystallized hydroxyapatite, confers stiffness to bone. ${ }^{23}$ The HA crystals, present in the form of plates or needles in the bone, are about 15-200 nm long, 10-80 nm wide, and 2-7 nm thick. ${ }^{24}$ Ideally, a biomaterial suitable for bone repair should be able to mimic the natural extracellular matrix. Therefore, we chose nHA as one of the main components to improve the hydrophilic nature of the COL/PCL/ nHA-SBF scaffold (Figure 2) and achieve osteoinductive

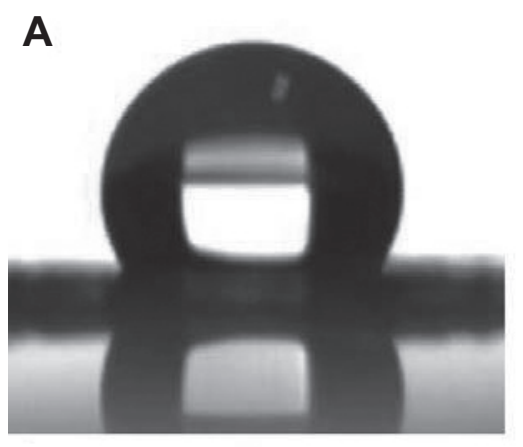

B

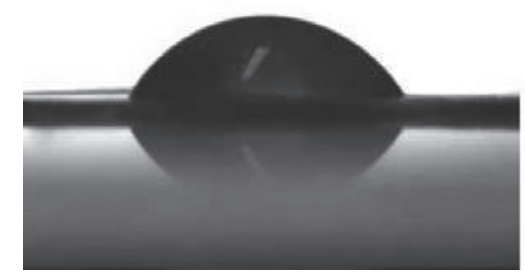

C

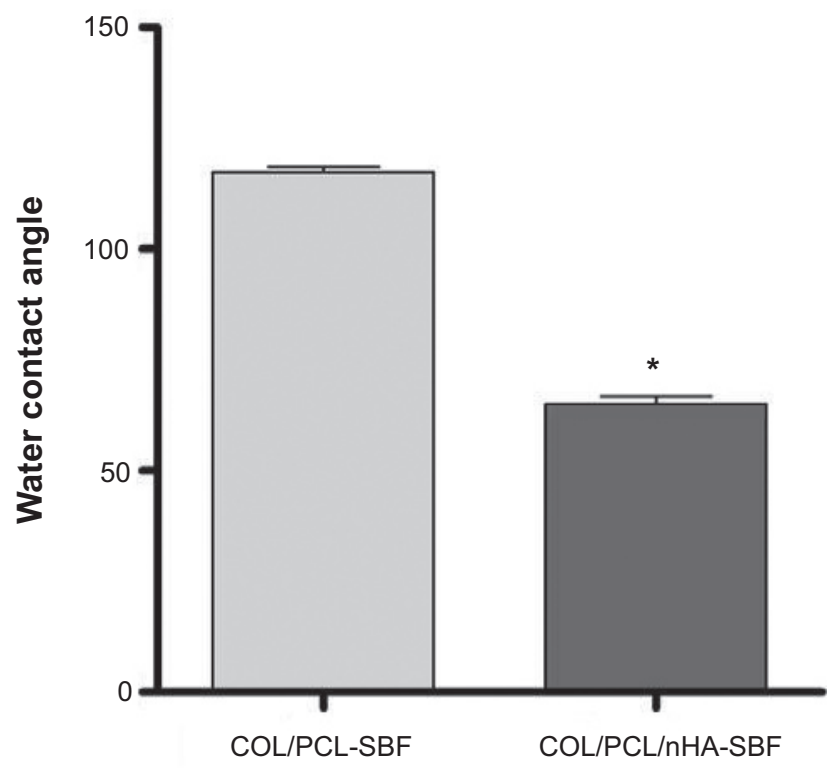

Figure 2 Water contact angles of COL/PCL-SBF, COL/PCL/nHA-SBF and their data presented as mean values \pm standard deviation.

Notes: The water contact angle of COL/PCL-SBF is about $117.35^{\circ}(\mathbf{A})$. The water contact angle of COL/PCL/nHA-SBF is about $67.43^{\circ}(\mathbf{B})$. Additionally, their statistical data presented as mean \pm standard deviation; $* \mathrm{P}<0.05$ (COL/PCL-SBF versus COL/PCL/nHA-SBF) (C).

Abbreviations: COL/PCL-SBF, the electrospun type-I collagen/ poly( $\varepsilon$-caprolactone) scaffold immersed in simulated body fluid; COL/PCL/nHA-SBF, the electrospun type-I collagen/ poly( $\varepsilon$-caprolactone)/ nanoscale hydroxyapatite scaffold immersed in simulated body fluid. 

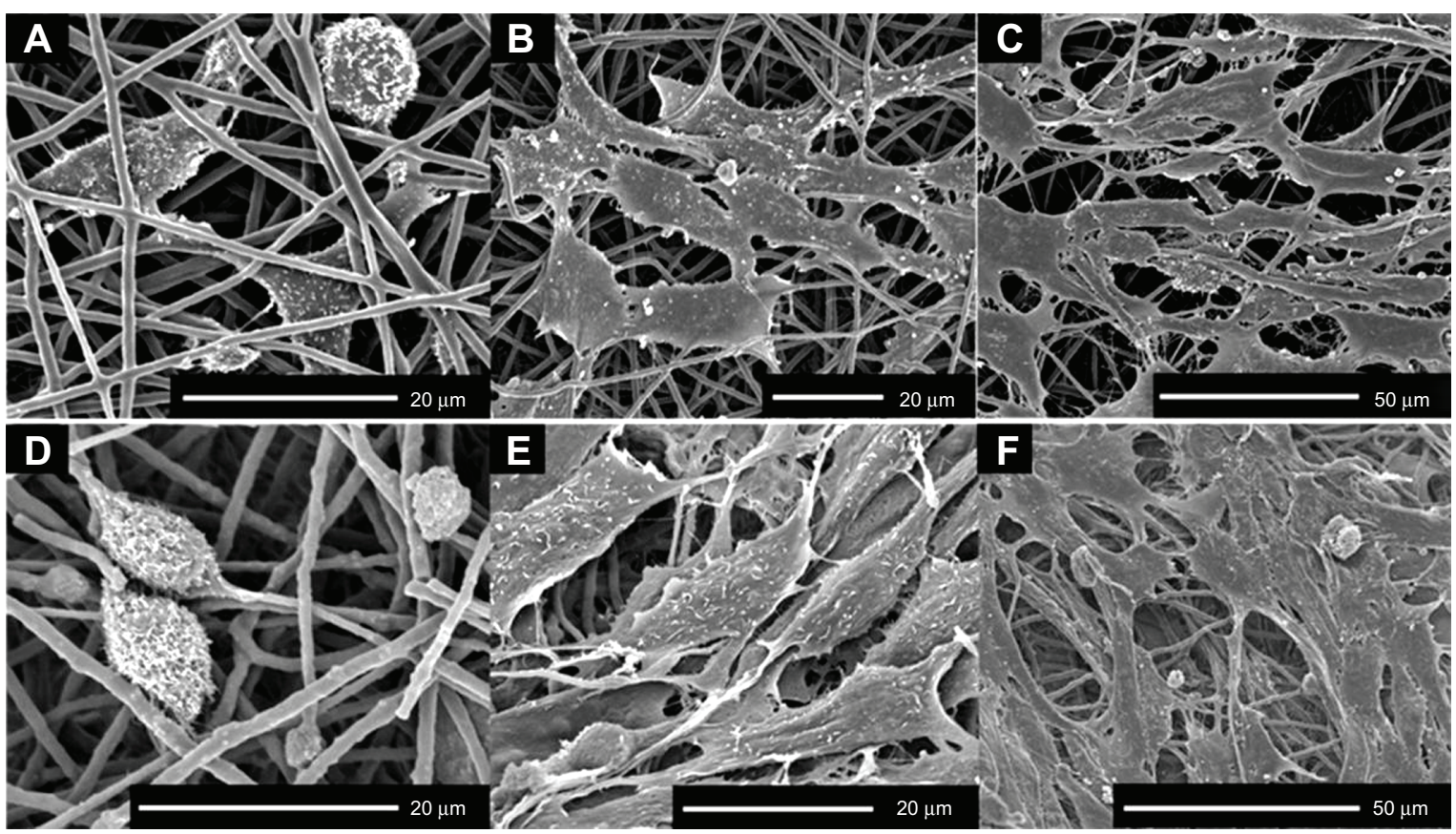

Figure 3 Scanning electron micrographs of PDLCs cultured on the COL/PCL-SBF and COL/PCL/nHA-SBF scaffolds.

Notes: Cells on the COL/PCL-SBF (A, B, C) and COL/PCL/nHA-SBF (D, E, F) appeared to have no significant difference in morphology. The cells adhered to the fibers were spindle-shaped on the first day (A, D), and then extended gradually and adequately at day 3 (B, E). At day 8, the PDLCs were further flattened and stretched out flopodial (C, F). Abbreviations: COL/PCL-SBF, the electrospun type-I collagen/ poly( $\varepsilon$-caprolactone) scaffold immersed in simulated body fluid; COL/PCL/nHA-SBF, the electrospun type-I collagen/ poly( $\varepsilon$-caprolactone)/ nanoscale hydroxyapatite scaffold immersed in simulated body fluid.

functions including cell adhesion, cell proliferation, synthesis of bone-related proteins, and deposition of calciumcontaining mineral.

Collagen, which can be considered as the matrix, exists in the form of small microfibers, the diameters of which range from 100 to 2,000 $\mathrm{nm}$. Collagen has properties conducive to tissue regeneration, including good biocompatibility, no immunogenicity, no toxicity, an ability to support and protect cells, and an ability to mediate cell adhesion. Once cells are inserted, growth may be able to continue as normal in the tissue. ${ }^{25-28}$ However, defects that limit the application of collagen include its rapid degradation, lack of mechanical properties, and poor stability. ${ }^{29}$ To overcome these problems, we introduced two further constituents, ie, genipin for crosslinking collagen and PCL to reform the properties of the scaffold. Genipin is a natural cross-linking agent extracted from the gardenia plant, and is much less cytotoxic than glutaraldehyde. Genipin can react with proteins and produce blue-colored fluorescent hydrogels, so as to achieve the purpose of cross-linking. ${ }^{30} \mathrm{PCL}$ is a semicrystalline, resorbable aliphatic polyester that has a much lower rate of degradation and excellent mechanical properties. ${ }^{31}$ Therefore, by including the effects of these two components, the composite scaffold can support integration, nutrient transfer, space maintenance, and manageability.
In alveolar bone, nHA deposits parallel to collagen fibers, and this may explain why a simple "rule of mixtures" approach cannot successfully explain the characteristics of bone. In order to mimic bone structure and composition, researchers have prepared COL/HA bone analog composites using a variety of conventional methods, including direct mineralization and chemical precipitation. ${ }^{32-38}$ In the present study, we used the technique of electrospinning to prepare a nanostructured fibrous composite to mimic

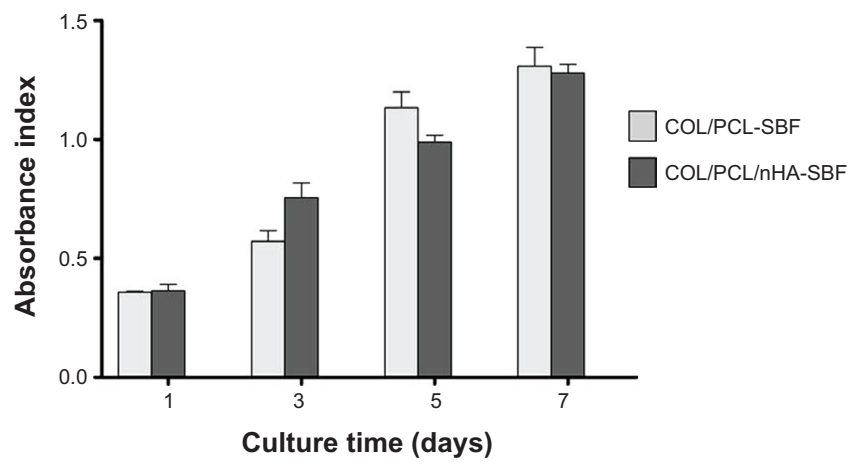

Figure 4 PDLC proliferation on the COL/PCL-SBF and COL/PCL/nHA-SBF scaffolds. Notes: Data represent mean \pm standard deviation. No significant difference against cell proliferation is shown on the two scaffolds (COL/PCL-SBF versus COL/PCL/ nHA-SBF).

Abbreviations: COL/PCL-SBF, the electrospun type-I collagen/ poly( $\varepsilon$-caprolactone) scaffold immersed in simulated body fluid; COL/PCL/nHA-SBF, the electrospun type-I collagen/ poly( $\varepsilon$-caprolactone)/ nanoscale hydroxyapatite scaffold immersed in simulated body fluid. 
A

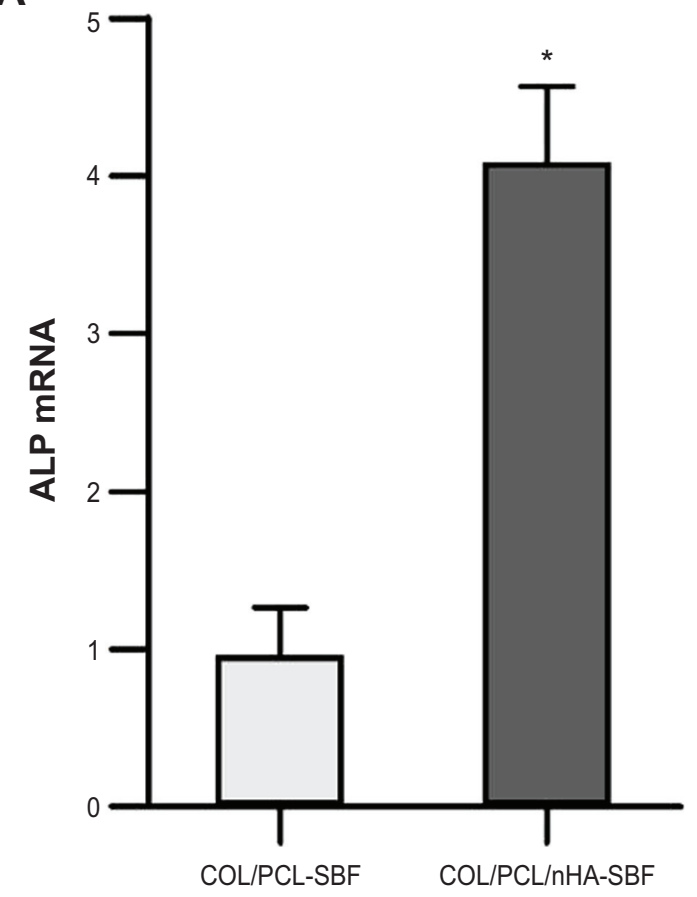

B

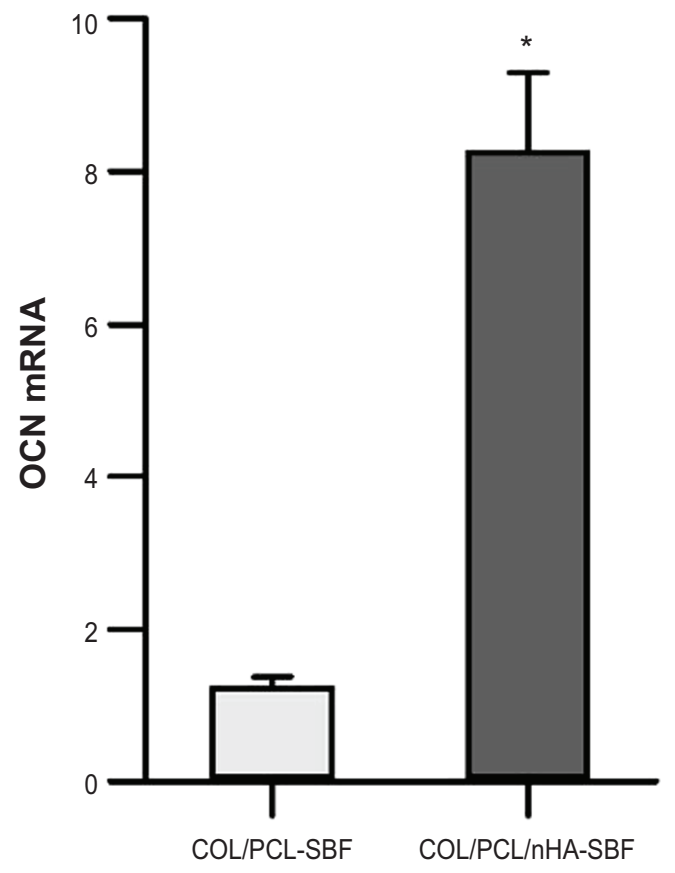

Figure 5 Real-time polymerase chain reaction analysis of ALP and OCN messenger ribonucleic acid expression in periodontal ligament cells on two scaffolds. Notes: Gene expression was normalized to housekeeping gene $\beta$-actin expression. Periodontal ligament cells were cultivated for I0 days on the COL/PCL-SBF and COL/ $\mathrm{PCL} / \mathrm{nHA}$-SBF scffolds. Data represents mean \pm standard deviation $(\mathrm{n}=3) ; * P<0.05$ (COL/PCL-SBF versus COL/PCL/nHA-SBF).

Abbreviations: ALP, phosphatase; OCN, osteocalcin, COL/PCL-SBF, the electrospun type-I collagen/ poly( $\varepsilon$-caprolactone) scaffold immersed in simulated body fluid; COL/ $\mathrm{PCL} / \mathrm{nHA}$-SBF, the electrospun type-I collagen/ poly( $\varepsilon$-caprolactone)/ nanoscale hydroxyapatite scaffold immersed in simulated body fluid.

bone structure as far as possible. This composite structure had a rough surface because of the nHA packed inside the organic fibers, and had a diameter of about $377 \mathrm{~nm}$ (Figure 1B). After incubation for 7 days in SBF, calcium phosphate was precipitated successfully, indicating the mineralization potential of this scaffold (Figure 1D). In the composite scaffold, the fibers consisting of COL and PCL can be considered as a load transfer medium that transfers the load to the intrinsically rigid nHA crystals. In addition, the nHA could "cross-link" the fibers through mechanical interlocking or by forming calcium ion bridges, thus having an osteoinductive function. ${ }^{39}$

The morphology and proliferation of cells on the scaffold are important evaluating indicators of the material. In this study, scanning electron microscopy and Cell Counting Kit-8 assays showed that PDLCs adhered to the scaffold extended adequately and proliferated stably. Confirmation of PDLC proliferation was also obtained by scanning electron microscopy (Figure 2). The PDLCs were capable of spanning adjacent fibers, so could create bridges between different layers. As a result, the PDLCs colonized the pores of the scaffold gradually until they were completely filled.

Biomineralization is an essential process in the formation of bone and teeth. We evaluated the mineralization ability of the scaffold by examining apatite formation on its surface after incubation in SBF for 7 days. Without pretreatment, hardly any apatite formation occurs spontaneously on most synthetic polymers. ${ }^{40-42}$ This is consistent with our finding that apatite deposited only on COL/PCL/nHA after incubation for 7 days; this can be attributed to the partial dissolution of nHA and subsequent release of calcium ions, which favors formation of apatite, and/or to the exposure of nHA particles on the COL/PCL surface, providing nucleation sites for apatite formation and growth. ${ }^{43}$ In addition, an appropriate calcium ion concentration also contributes to the proliferation and differentiation of cells by changing the expression of specific calcium ion channel isoforms.

Calcium ion concentrations reportedly exceed physiological concentrations at sites of bone erosion, reaching as high as $40 \mathrm{mM}$. In addition, higher calcium ion concentrations could promote differentiation of osteoblast and benefit bone mineralization. ${ }^{44}$ In the initial stage of mineralization, formation of HA crystals occurs inside the cells. The HA then propagates into the extracellular matrix and deposits between collagen fibers. ALP subsequently promotes mineralization by hydrolyzing pyrophosphate and providing inorganic phosphate. ${ }^{45}$ Therefore, ALP is a widely recognized marker of early osteoblastic differentiation. It was also found that the 
ALP activity in the osteoblast-like cells on the apatite/collagen composite coating was significantly higher than that on the apatite coating. This could be attributed to the collagen in the coating, which has been shown to have a positive effect on the ALP activity of osteoblasts. ${ }^{46}$ Osteocalcin, a major noncollagenous protein component of bone extracellular matrix, is synthesized and secreted exclusively by osteoblasts in the late stage of maturation, and is considered to be an indicator of osteoblast differentiation. Osteocalcin is believed to play an active role in controlling nucleation of HA crystals. ${ }^{47}$ In this study, PDLCs could make contact with calcium phosphate directly and early on in the growth process, and be influenced by the osteoinductive potential of the scaffold. With increasing culture time, PCL and COL would gradually degrade, enabling the nanoscale calcium phosphate compound and nHA to be released and continue the osteoinductive effect. Therefore, on day 10 of coculture, expression of ALP and osteocalcin in PDLCs from the COL/PCL/nHA-SBF group was higher than in the control group, indicating that the experimental scaffold has the potential to induce osteogenic differentiation of preosteoblasts in PDLCs.

There are three main stages in modification of gene expression, ie, proliferation, maturation of the extracellular matrix, and mineralization. The peaks of these three stages cannot occur at the same time, and cells can passage through but not pass to the next stage without further signals. ${ }^{48,49}$ That is to say, once the modifications in gene expression are induced, the rate of cell proliferation may gradually get into the deceleration period. Therefore, in this study, although PDLCs could proliferate stably on the COL/PCL/nHA-SBF scaffold, there was no significant difference in cell numbers between the groups over 7 days of culture, which may be the result of earlier cell differentiation on the composite surface.

\section{Conclusion}

In this study, we adopted the method of electrospinning followed by biomimetic mineralization to produce a COL/PCL/ nHA-SBF scaffold composed of electrospun fibers containing nHA. The scaffold obtained had good biocompatiblity and osteoinductive ability, and may have a role in periodontal tissue engineering.

\section{Acknowledgments}

This work was supported by grants from the National Natural Science Foundation $(81271155,81300852)$, the Key Project of Science and Technology Bureau of Jiangsu Province (BL2013002), the Jiangsu Province Natural Science Foundation of China (BK20130079), and the Nanjing Science and
Technology Development Foundation (201303022). The authors thank Professor Zhigang Zou (Nanjing University) for his critical support and assistance with the project design.

\section{Disclosure}

The authors report no conflicts of interest in this work.

\section{References}

1. Egelberg J. Regeneration and repair of periodontal tissues. J Periodontal Res. 1987;22(3):233-242.

2. Ivanovski S. Periodontal regeneration. Aust Dent J. 2009;54 Suppl 1: S118-S128.

3. Carlo Reis EC, Borges AP, Araújo MV, Mendes VC, Guan L, Davies JE. Periodontal regeneration using a bilayered PLGA/calcium phosphate construct. Biomaterials. 2011;32(35):9244-9253.

4. Rios HF, Lin Z, Oh B, Park CH, Giannobile WV. Cell-and gene-based therapeutic strategies for periodontal regenerative medicine. $J$ Periodontol. 2011;82(9):1223-1237.

5. Koop R, Merheb J, Quirynen M. Periodontal regeneration with enamel matrix derivative in reconstructive periodontal therapy: a systematic review. J Periodontol. 2012;83(6):707-720.

6. Kruse A, Jung R, Nicholls F, Zwahlen R, Hämmerle C, Weber F. Bone regeneration in the presence of a synthetic hydroxyapatite/silica oxidebased and a xenogenic hydroxyapatite-based bone substitute material. Clin Oral Implants Res. 2011;22(5):506-511.

7. Costantino PD, Friedman CD. Synthetic bone graft substitutes. Otolaryngol Clin North Am. 1994;27(5):1037-1074.

8. Giannoudis PV, Dinopoulos H, Tsiridis E. Bone substitutes: an update. Injury. 2005;36(3):S20-S27.

9. Hannink G, Arts J. Bioresorbability, porosity and mechanical strength of bone substitutes: what is optimal for bone regeneration? Injury. 2011;42:S22-S25.

10. Petite H, Viateau V, Bensaid W, et al. Tissue-engineered bone regeneration. Nat Biotechnol. 2000;18(9):959-963.

11. Suchanek W, Yoshimura M. Processing and properties of hydroxyapatite-based biomaterials for use as hard tissue replacement implants. J Mater Res. 1998;13(01):94-117.

12. Bertazzo S, Bertran C. Morphological and dimensional characteristics of bone mineral crystals. Key Eng Mater. 2006;309:3-6.

13. Zhang ZG, Li ZH, Mao XZ, Wang WC. Advances in bone repair with nanobiomaterials: mini-review. Cytotechnology. 2011;63(5): 437-443.

14. Sun W, Chu C, Wang J, Zhao H. Comparison of periodontal ligament cells responses to dense and nanophase hydroxyapatite. J Mater Sci Mater Med. 2007;18(5):677-683.

15. Bhardwaj N, Kundu SC. Electrospinning: a fascinating fiber fabrication technique. Biotechnol Adv. 2010;28(3):325-347.

16. Wang X, Ding B, Li B. Biomimetic electrospun nanofibrous structures for tissue engineering. Mater Today. 2013;16(6):229-241.

17. Chen R, Hunt JA. Biomimetic materials processing for tissue-engineering processes. J Mater Chem. 2007;17(38):3974-3979.

18. Wang J, Yu X. Preparation, characterization and in vitro analysis of novel structured nanofibrous scaffolds for bone tissue engineering. Acta Biomater. 2010;6(8):3004-3012.

19. Liu W, Thomopoulos S, Xia Y. Electrospun nanofibers for regenerative medicine. Adv Healthc Mater. 2012;1(1):10-25.

20. Kokubo T, Takadama $\mathrm{H}$. How useful is SBF in predicting in vivo bone bioactivity? Biomaterials. 2006;27(15):2907-2915.

21. Kokubo T, Hanakawa M, Kawashita M, et al. Apatite formation on non-woven fabric of carboxymethylated chitin in SBF. Biomaterials. 2004;25(18):4485-4488.

22. Livak KJ, Schmittgen TD. Analysis of relative gene expression data using real-time quantitative PCR and the 29-Delta Delta C(T) method. Methods. 2001;25(4):402-408. 
23. Xu Q, Lu H, Zhang J, Lu G, Deng Z, Mo A. Tissue engineering scaffold material of porous nanohydroxyapatite/polyamide 66. Int J Nanomedicine. 2010;5:331-335.

24. Fratzl P, Gupta H, Paschalis E, Roschger P. Structure and mechanical quality of the collagen - mineral nano-composite in bone. J Mater Chem. 2004;14(14):2115-2123.

25. Oliveira SM, Ringshia RA, Legeros RZ, et al. An improved collagen scaffold for skeletal regeneration. J Biomed Mater Res A. 2010; 94(2):371-379.

26. Gómez-Guillén M, Giménez B, López-Caballero M, Montero M. Functional and bioactive properties of collagen and gelatin from alternative sources: a review. Food Hydrocoll. 2011;25(8):1813-1827.

27. Snibson GR. Collagen cross-linking: a new treatment paradigm in corneal disease - a review. Clin Experiment Ophthalmol. 2010;38(2): 141-153.

28. Stoecklin-Wasmer C, Rutjes A, da Costa B, Salvi G, Jüni P, Sculean A. Absorbable collagen membranes for periodontal regeneration: a systematic review. J Dent Res. 2013;92(9):773-781.

29. Behring J, Junker R, Walboomers XF, Chessnut B, Jansen JA. Toward guided tissue and bone regeneration: morphology, attachment, proliferation, and migration of cells cultured on collagen barrier membranes. A systematic review. Odontology. 2008;96(1):1-11.

30. Kuo YC, Ku IN. Cartilage regeneration by novel polyethylene oxide/ chitin/chitosan scaffolds. Biomacromolecules. 2008;9(10):2662-2669.

31. Gentile P, Chiono V, Tonda, Turo C, Ferreira AM, Ciardelli G. Polymeric membranes for guided bone regeneration. Biotechnol J. 2011; 6(10):1187-1197.

32. Wahl D, Czernuszka J. Collagen-hydroxyapatite composites for hard tissue repair. Eur Cell Mater. 2006;11:43-56.

33. Itoh S, Kikuchi M, Takakuda K, et al. The biocompatibility and osteoconductive activity of a novel hydroxyapatite/collagen composite biomaterial, and its function as a carrier of rhBMP-2. J Biomed Mater Res. 2001;54(3):445-453.

34. Tampieri A, Celotti G, Landi E, Sandri M, Roveri N, Falini G. Biologically inspired synthesis of bone-like composite: self-assembled collagen fibers/hydroxyapatite nanocrystals. J Biomed Mater Res A. 2003;67(2):618-625.

35. Roveri N, Falini G, Sidoti M, et al. Biologically inspired growth of hydroxyapatite nanocrystals inside self-assembled collagen fibers. Mater Sci Eng C. 2003;23(3):441-446.

36. Kikuchi M, Itoh S, Ichinose S, Shinomiya K, Tanaka J. Self-organization mechanism in a bone-like hydroxyapatite/collagen nanocomposite synthesized in vitro and its biological reaction in vivo. Biomaterials. 2001;22(13):1705-1711.
37. Chang MC, Ikoma T, Kikuchi M, Tanaka J. Preparation of a porous hydroxyapatite/collagen nanocomposite using glutaraldehyde as a crosslinkage agent. J Mater Sci Lett. 2001;20(13):1199-1201.

38. Yamauchi K, Goda T, Takeuchi N, Einaga H, Tanabe T. Preparation of collagen/calcium phosphate multilayer sheet using enzymatic mineralization. Biomaterials. 2004;25(24):5481-5489.

39. Hellmich C, Ulm FJ. Are mineralized tissues open crystal foams reinforced by crosslinked collagen? Some energy arguments. J Biomech. 2002;35(9):1199-1212.

40. Chen J, Chu B, Hsiao BS. Mineralization of hydroxyapatite in electrospun nanofibrous poly(L-lactic acid) scaffolds. J Biomed Mater Res A. 2006;79(2):307-317.

41. Oyane A, Uchida M, Choong C, Triffitt J, Jones J, Ito A. Simple surface modification of poly(epsilon-caprolactone) for apatite deposition from simulated body fluid. Biomaterials. 2005;26(15):2407-2413.

42. Oyane A, Uchida M, Yokoyama Y, Choong C, Triffitt J, Ito A. Simple surface modification of poly(epsilon-caprolactone) to induce its apatiteforming ability. J Biomed Mater Res A. 2005;75(1):138-145.

43. Yang F, Both SK, Yang X, Walboomers XF, Jansen JA. Development of an electrospun nano-apatite/PCL composite membrane for GTR/ GBR application. Acta Biomater. 2009;5(9):3295-3304.

44. Maeno S, Niki Y, Matsumoto H, et al. The effect of calcium ion concentration on osteoblast viability, proliferation and differentiation in monolayer and 3D culture. Biomaterials. 2005;26(23):4847-4855.

45. Orimo $\mathrm{H}$. The mechanism of mineralization and the role of alkaline phosphatase in health and disease. J Nippon Med Sch. 2010;77(1):4-12.

46. Chen Y, Mak A, Wang M, Li J, Wong M. In vitro behavior of osteoblastlike cells on PLLA films with a biomimetic apatite or apatite/collagen composite coating. J Mater Sci Mater Med. 2008;19(6):2261-2268.

47. Sila-Asna M, Bunyaratvej A, Maeda S, Kitaguchi H, Bunyaratavej N. Osteoblast differentiation and bone formation gene expression in strontium-inducing bone marrow mesenchymal stem cell. Kobe J Med Sci. 2007;53(1-2):25-35.

48. Stein GS, Lian JB, Owen TA. Relationship of cell growth to the regulation of tissue-specific gene expression during osteoblast differentiation. FASEB J. 1990;4(13):3111-3123.

49. Malaval L, Liu F, Roche P, Aubin JE. Kinetics of osteoprogenitor proliferation and osteoblast differentiation in vitro. $J$ Cell Biochem. 1999;74(4):616-627.
International Journal of Nanomedicine

\section{Publish your work in this journal}

The International Journal of Nanomedicine is an international, peerreviewed journal focusing on the application of nanotechnology in diagnostics, therapeutics, and drug delivery systems throughout the biomedical field. This journal is indexed on PubMed Central, MedLine, CAS, SciSearch $®$, Current Contents $\AA /$ Clinical Medicine,

\section{Dovepress}

Journal Citation Reports/Science Edition, EMBase, Scopus and the Elsevier Bibliographic databases. The manuscript management system is completely online and includes a very quick and fair peer-review system, which is all easy to use. Visit http://www.dovepress.com/ testimonials.php to read real quotes from published authors. 